\title{
Continuous-Time Filter Design Optimized for Reduced Die Area
}

\author{
Charles Myers, Student Member, IEEE, Brandon Greenley, Member, IEEE, Daniel Thomas, Member, IEEE, and \\ Un-Ku Moon, Senior Member, IEEE
}

\begin{abstract}
A method for distributing capacitor and resistor area to optimally reduce die area in a given continuous-time filter design while maintaining the filter's designed signal-to-noise ratio (SNR), frequency response, and topology is discussed. To do this, a basic linear programming algorithm is developed from derived circuit cost and constraint functions. Among the three major filter types of fifth-order under comparison, maximum combined capacitor and resistor die area savings of $21 \%$ results for the Butterworth filter. An alternative approach where the SNR is optimized for a given die area is also presented. Maximum improvement of the fifth-order Butterworth filter for this approach is $1.2 \mathrm{~dB}$.
\end{abstract}

Index Terms-Constraint and cost functions, continous-time filter, linear programming, maximize SNR, minimize die area, optimization.

\section{INTRODUCTION}

$\mathbf{T}$ HE reduction of die area for analog filter designs usually implies some kind of tradeoff. The most common of these is an increase in noise. This is especially true for switched-capacitor circuits where noise is dominated by $k T / C$. This situation is also true for continuous-time (CT) filters.

In most CT filters, capacitors contribute much more to the die area than resistors. The design of these filters is based on the placement of poles and zeros in the $s$-plane. These poles and zeros are directly correlated with circuit $R C$ time constants and define the operation of the filter. Any changes in capacitor or resistor value must be balanced by appropriate adjustment of other components to maintain the same filter frequency response.

Because capacitor area dominates resistor area, die area reduction requires a decrease in capacitor value, causing a required increase in resistor value. This increase in resistor value translates to an increase in overall noise and thereby degrades the signal-to-noise ratio (SNR). The proposed design method finds an optimal distribution of capacitor and resistor area, which reduces the die area required for a filter design of a given frequency response, filter topology, and SNR.

The proposed optimization method for minimizing capacitor and resistor area for a given filter design begins with the following consideration. Higher order CT filters have several stages, each with an associated capacitor that can be scaled independently of the other stages. This increases or decreases the

Manuscript received February 20, 2003; revised August 27, 2003. This paper was recommended by Associate Editor A. Petraglia.

C. Myers and U. Moon are with the Department of Electrical and Computer Engineering, Oregon State University, Corvallis, OR 97331-3211 USA (e-mail: moon@ece.orst.edu).

B. Greenley is with Tektronix, Inc., Beaverton, OR 97077 USA.

D. Thomas is with Network Elements, Beaverton, OR 97006 USA.

Digital Object Identifier 10.1109/TCSII.2003.822425 filter noise at the output due to the required complementary scaling of resistor values within that stage. Because each resistor sees a different gain to the output, the change in noise may be different for scaling different capacitors. It follows that there is a minimum die area that can be achieved for a given SNR design requirement, and conversely, there is some distribution of a fixed die area that maximizes SNR for a given area budget [1]. A simple linear programming algorithm can be used to find such an optimum without altering the filter's frequency response or the topology/structure.

The linear programming approach to filter optimization is not new. One such prior art on this topic has shown enhanced time-domain and frequency-domain filter responses in digital filters by optimally placing pole/zero locations [2]. Other techniques for optimum pole/zero placement have also been performed for analog filters [3]. Some optimization techniques involve state-space methods targeting reduced filter sensitivity [4], and pre-design optimization of bandpass filters that determines the realization feasibility of a filter design for given level of distortion and dynamic range [5]. Our linear programming approach presented here is unique in that it preserves the filter responses and topology ( $L C$ ladder example is used) while targeting efficient integrated circuit (IC) implementation. It is possible to apply this optimization technique in addition to other techniques, specifically [3], since our optimization process does not change the filter transfer function. The proposed linear programming optimization process either minimizes the die area while keeping the SNR/noise of the filter unchanged, or maximizes the SNR (minimizing noise) while keeping the IC die area fixed. A similar goal in the context of switched-capacitor filters has produced closed-form expressions for minimizing total capacitance in biquads [6]. However, the results do not stem from linear programming but from mathematical derivations of a few specific biquads given a set of approximations.

An optimization concept for maximizing the SNR of a given filter design was introduced in [1], which included a simplified description of capacitor area consideration using linear programming techniques. A complete description of our optimization algorithm will be presented here. The algorithm can target optimization of either die area or SNR, including the area contributions of both capacitors and resistors.

Sections II-V will include discussions on the derived filter structure that is used as an example in the proposed optimization (Section II), the development of a constant-SNR optimization algorithm (Section III), the development of a constant-area optimization algorithm (Section IV), the SPICE simulated results (Section V), and the conclusions. 


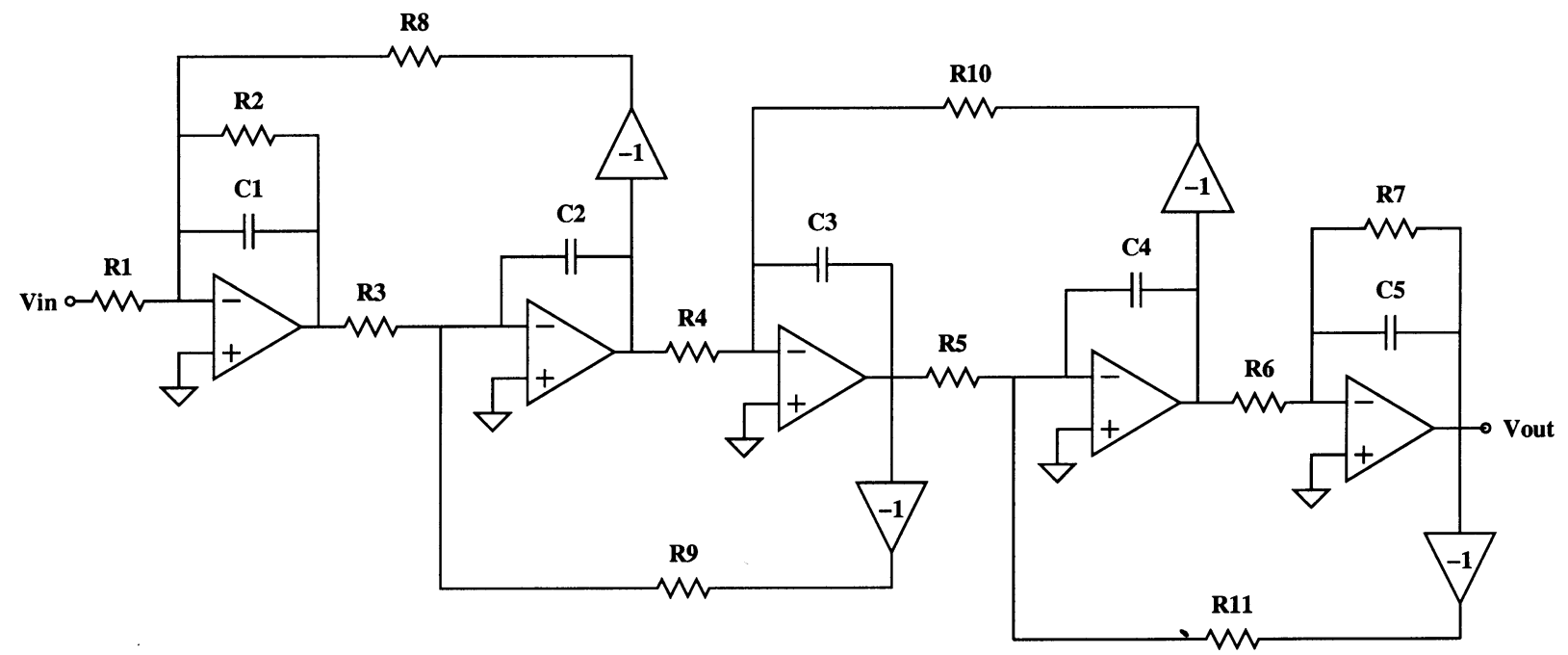

Fig. 1. Fifth-order filter circuit structure.

TABLE I

NORMALIZED FIFTH-ORdER FILTER COEFFICIENTS

\begin{tabular}{c||c|c|c}
\hline Component & Bessel & Chebychev & Butterworth \\
\hline \hline$R_{1}-R_{11}$ & 1.0 & 1.0 & 1.0 \\
\hline$C_{1}$ & 0.1743 & 1.8068 & 0.6180 \\
\hline$C_{2}$ & 0.5072 & 1.3025 & 1.6180 \\
\hline$C_{3}$ & 0.8040 & 2.6917 & 2.0000 \\
\hline$C_{4}$ & 1.1110 & 1.3025 & 1.6180 \\
\hline$C_{5}$ & 2.2582 & 1.8068 & 0.6180 \\
\hline
\end{tabular}

\section{Fifth-ORder Test Filter}

The active filter circuit model used for each of the filters tested was derived from a standard (doubly terminated) $L C$ ladder configuration. The fifth-order $L C$ ladder was then transformed by mathematical manipulation into an active $R C$ circuit made up of integrator blocks (Fig. 1). This process is outlined in the literature [9]. Our choice of filter type and topology/structure is somewhat arbitrary and was based on "popularity" as most circuit designers choose the filter type and topology based on application requirements or sometimes due to other reasons as simple as personal preference. The proposed design technique for reducing the die area of a CT filter will generally apply to all integrator-based active filter structures (e.g., standard $L C$ ladder, various biquads, orthonormal filters [7], etc.). The linear optimization algorithm discussed in Section III is applied to three different filters: Bessel, Chebychev (Type I, 0.5-dB passband ripple), and Butterworth filters. All three filters are low pass and have a corner frequency equal to $22 \mathrm{kHz}$. It is important to note that use of this optimization is not limited to the design of audio or audio range filters. The optimization is not affected by the choice of filter corner frequency.

There is no difference in circuit structure for each of the three filters as all can be derived from the same $L C$ ladder configuration. The only differences are the specific component values chosen. The component values (Table I) can be found in any of several filter books [8]-[10]. After assigning the component values, three scaling operations must take place. The first scales
TABLE II

Node-Voltage Scaled Filter Component Values

\begin{tabular}{c||c|c|c}
\hline Component & Bessel & Chebychev & Butterworth \\
\hline \hline$C_{1}$ & $15.493 \mathrm{pF}$ & $126.36 \mathrm{pF}$ & $53.133 \mathrm{pF}$ \\
\hline$C_{2}$ & $47.955 \mathrm{pF}$ & $132.62 \mathrm{pF}$ & $117.99 \mathrm{pF}$ \\
\hline$C_{3}$ & $75.652 \mathrm{pF}$ & $185.17 \mathrm{pF}$ & $113.83 \mathrm{pF}$ \\
\hline$C_{4}$ & $86.293 \mathrm{pF}$ & $110.67 \mathrm{pF}$ & $77.163 \mathrm{pF}$ \\
\hline$C_{5}$ & $99.949 \mathrm{pF}$ & $80.141 \mathrm{pF}$ & $27.413 \mathrm{pF}$ \\
\hline \hline$R_{1}$ & $81.500 \mathrm{k} \Omega$ & $81.500 \mathrm{k} \Omega$ & $81.500 \mathrm{k} \Omega$ \\
\hline$R_{2}$ & $81.537 \mathrm{k} \Omega$ & $103.45 \mathrm{k} \Omega$ & $84.143 \mathrm{k} \Omega$ \\
\hline$R_{3}$ & $81.537 \mathrm{k} \Omega$ & $103.45 \mathrm{k} \Omega$ & $84.143 \mathrm{k} \Omega$ \\
\hline$R_{4}$ & $76.477 \mathrm{k} \Omega$ & $71.052 \mathrm{k} \Omega$ & $99.203 \mathrm{k} \Omega$ \\
\hline$R_{5}$ & $76.920 \mathrm{k} \Omega$ & $105.15 \mathrm{k} \Omega$ & $127.11 \mathrm{k} \Omega$ \\
\hline$R_{6}$ & $93.123 \mathrm{k} \Omega$ & $85.151 \mathrm{k} \Omega$ & $151.75 \mathrm{k} \Omega$ \\
\hline$R_{7}$ & $163.08 \mathrm{k} \Omega$ & $163.10 \mathrm{k} \Omega$ & $163.09 \mathrm{k} \Omega$ \\
\hline$R_{8}$ & $76.477 \mathrm{k} \Omega$ & $71.052 \mathrm{k} \Omega$ & $99.202 \mathrm{k} \Omega$ \\
\hline$R_{9}$ & $76.920 \mathrm{k} \Omega$ & $105.15 \mathrm{k} \Omega$ & $127.11 \mathrm{k} \Omega$ \\
\hline$R_{10}$ & $93.123 \mathrm{k} \Omega$ & $85.151 \mathrm{k} \Omega$ & $151.75 \mathrm{k} \Omega$ \\
\hline$R_{11}$ & $163.08 \mathrm{k} \Omega$ & $163.10 \mathrm{k} \Omega$ & $163.09 \mathrm{k} \Omega$ \\
\hline
\end{tabular}

the corner frequency from unity (1 radian per second) to the desired value, the second scales the components to usable values for realization, and the third is the node-voltage scaling which improves the dynamic range of active filters [10]. This effectively scales the peak gain at each output of the operational amplifier in order to use its full output range. The node-voltage scaled component values for each of the three filters are shown in Table II. These component values are the starting values for all filter optimizations in Sections III-V.

\section{CONSTANT-SNR LINEAR OPTIMIZATION ALGORITHM}

In order to use any linear programming method, such as Karmarkar's Algorithm [11], a set of linear cost and constraint functions must be derived. For the optimization proposed in this section, die area should be minimized while maintaining a fixed SNR. In high-SNR filters, die area is dominated by the capacitors which have area densities near $1 \mathrm{fF} / \mu \mathrm{m}^{2}$. Resistors can have area densities ranging from $10 \Omega / \mu \mathrm{m}^{2}$ to around 
$1 \mathrm{k} \Omega / \mu \mathrm{m}^{2}$. For improved accuracy over the optimization done in [1], both capacitor and resistor area is included in the derivation of the cost and constraint functions.

Before linear cost and constraint functions can be derived, it is necessary to define some relationships between component values within the circuit. There are several relationships that must not change if the frequency response of the filter is to be preserved. The first of these is the $R C$ time constant associated with each of the capacitors $C_{1}-C_{5}$ (refer to Fig. 1):

$$
\begin{array}{ll}
\tau_{1}=C_{1} R_{1}, & \tau_{2}=C_{2} R_{3}, \quad \tau_{3}=C_{3} R_{4}, \\
\tau_{4}=C_{4} R_{5}, & \tau_{5}=C_{5} R_{6} .
\end{array}
$$

The second is the ratio between resistors setting the closed loop gains at the input of each integrator circuit:

$$
\begin{array}{cl}
k_{2}=\frac{R_{2}}{R_{1}}, & k_{8}=\frac{R_{8}}{R_{1}}, \quad k_{9}=\frac{R_{9}}{R_{3}}, \\
k_{10}=\frac{R_{10}}{R_{4}}, & k_{11}=\frac{R_{11}}{R_{5}}, \quad k_{7}=\frac{R_{7}}{R_{6}} .
\end{array}
$$

With these two sets of equations (1) and (2), it can be seen that all components can be written in terms of the five opamp input resistors $R_{1}, R_{3}, R_{4}, R_{5}$, and $R_{6}$.

The constraint of our linear programming algorithm is SNR. For a fixed and defined input range, constant SNR is equivalent to constant noise. The output referred noise power for the filter can be expressed as

$$
\text { Noise } \propto \sum_{i=1}^{11} R_{i} \int_{\mathrm{BW}} A_{i}^{2}(f) d f=\sum_{i=1}^{11} R_{i} E_{i}
$$

where $A_{i}$ is the gain (magnitude frequency response) from $R_{i}$ to the output. ${ }^{1}$

This is not the exact noise value, but is proportional to the actual noise, which is in the form $4 k T R A^{2}$. Since we are only interested in maintaining the designed noise level, this information is sufficient. The gain is integrated over a bandwidth range, the noise bandwidth, and is the effective targeted range of the optimization. For many applications, the noise bandwidth would be equal to the corner frequency of the filter.

The cost function is the equation describing the area consumed by the capacitors and resistors. Written in its most basic form

$$
\begin{aligned}
\text { Area }= & \left(C_{1}+C_{2}+C_{3}+C_{4}+C_{5}\right)\left(C_{-} \text {Density }^{-1}\right) \\
& +\left(R_{1}+R_{2}+\cdots+R_{10}+R_{11}\right)\left(R_{\text {_Density }}{ }^{-1}\right) .
\end{aligned}
$$

The application of (1) and (2) to the constraint and cost functions (3) and (4), replacing $R_{1}, R_{3}, R_{4}, R_{5}$, and $R_{6}$ with $x_{1}-x_{5}$, results in the constraint equation

Noise $\propto$ constraint

$$
\begin{aligned}
& =x_{1}\left(E_{1}+k_{2} E_{2}+k_{8} E_{8}\right)+x_{2}\left(E_{3}+k_{9} E_{9}\right) \\
& +x_{3}\left(E_{4}+k_{10} E_{10}\right)+x_{4}\left(E_{5}+k_{11} E_{11}\right) \\
& +x_{5}\left(E_{6}+k_{7} E_{7}\right)
\end{aligned}
$$

${ }^{1}$ Only resistors are considered here. Inclusion of opamp noise contribution would yield more precise optimization results. and a cost equation of

$$
\begin{aligned}
\text { Area }=\text { cost }= & \frac{\tau_{1}}{x_{1}\left(C \_ \text {Density }\right)}+\frac{x_{1}\left(1+k_{2}+k_{8}\right)}{\left(R \_ \text {Density }\right)} \\
& +\frac{\tau_{2}}{x_{2}\left(C \_ \text {Density }\right)}+\frac{x_{2}\left(1+k_{9}\right)}{\left(R \_ \text {Density }\right)} \\
& +\frac{\tau_{3}}{x_{3}\left(C \_ \text {Density }\right)}+\frac{x_{3}\left(1+k_{10}\right)}{\left(R \_ \text {Density }\right)} \\
& +\frac{\tau_{4}}{x_{4}\left(C \_ \text {Density }\right)}+\frac{x_{4}\left(1+k_{11}\right)}{\left(R \_ \text {Density }\right)} \\
& +\frac{\tau_{5}}{x_{5}\left(C \_ \text {Density }\right)}+\frac{x_{5}\left(1+k_{7}\right)}{\left(R \_ \text {Density }\right)} .
\end{aligned}
$$

These equations are written completely in terms of the constants defined in (1) and (2) and the five opamp resistor inputs $R_{1}, R_{3}$, $R_{4}, R_{5}$, and $R_{6}$.

Before illustrating the details of a chosen optimization method in the following, it is important to recognize that the given constraint and the cost equations (5) and (6) describe the main purpose of this work. Even though we arrive at this point with a simple set of derivations, it is the formulation of these two constraint and cost equations that allow us to find the optimal component values that will minimize the die area of the filter for a given SNR (or maximize SNR for a given die area, as discussed in Section IV).

In order to apply a linear programming method to these functions, they must all be linear. The constraint function (5) is linear, however, the cost function (6) is not. A simple solution is to complete a first-order Taylor Expansion on the cost function. This results in the following equation:

$$
\begin{aligned}
\text { cost } \simeq & x_{1}\left[\frac{-\tau_{1}}{x_{1(0)}^{2}\left(C \_ \text {Density }\right)}+\frac{1+k_{2}+k_{8}}{R \_ \text {Density }}\right] \\
& +\frac{2 \tau_{1}}{x_{1(0)}\left(C \_ \text {Density }\right)} \\
& +x_{2}\left[\frac{-\tau_{2}}{x_{2(0)}^{2}\left(C \_ \text {Density }\right)}+\frac{1+k_{9}}{R \_ \text {Density }}\right] \\
& +\frac{2 \tau_{2}}{x_{2(0)}\left(C \_ \text {Density }\right)} \\
& +x_{3}\left[\frac{-\tau_{3}}{x_{3(0)}^{2}\left(C \_ \text {Density }\right)}+\frac{1+k_{10}}{R \_ \text {Density }}\right] \\
& +\frac{2 \tau_{3}}{x_{3(0)}\left(C \_ \text {Density }\right)} \\
& +x_{4}\left[\frac{-\tau_{4}}{x_{4(0)}^{2}\left(C \_ \text {Density }\right)}+\frac{1+k_{11}}{R \_ \text {Density }}\right] \\
& +\frac{2 \tau_{4}}{x_{4(0)}\left(C \_ \text {Density }\right)} \\
& +x_{5}\left[\frac{-\tau_{5}}{x_{5(0)}^{2}\left(C \_ \text {Density }\right)}+\frac{1+k_{7}}{R \_ \text {Density }}\right] \\
& +\frac{2 \tau_{5}}{x_{5(0)}\left(C \_ \text {Density }\right)}
\end{aligned}
$$

where $\vec{x}_{(0)}$ is the initial condition about which the Taylor Series is expanded. 
With linear constraint and cost functions derived, it is now possible to apply a linear programming method to determine the optimum component area distribution. A modified Karmarkar's Algorithm is used. It is important to note here that any optimization scheme would result in an identical optimization given the same constraint and cost functions. Our choice of optimization method here is intended to provide a straightforward illustration of the proposed concept.

The forms of the constraint and cost equations for Karmarkar's Algorithm are the following [11]:

$$
\mathbf{A} \vec{x}=\text { constraint } \quad \text { and } \quad \vec{c}^{T} \vec{x}+D=\text { cost }
$$

where

$$
\begin{aligned}
& \mathbf{A}^{T}=\left[\begin{array}{c}
E_{1}+k_{2} E_{2}+k_{8} E_{8} \\
E_{3}+k_{9} E_{9} \\
E_{4}+k_{10} E_{10} \\
E_{5}+k_{11} E_{11} \\
E_{6}+k_{7} E_{7}
\end{array}\right]
\end{aligned}
$$

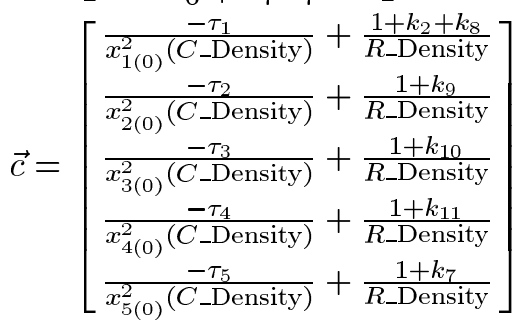

$$
\begin{aligned}
& \vec{x}^{T}=\left[\begin{array}{lllll}
x_{1} & x_{2} & x_{3} & x_{4} & x_{5}
\end{array}\right] \\
& D=\frac{2 \tau_{1}}{x_{1(0)}\left(C \_ \text {Density }\right)}+\frac{2 \tau_{2}}{x_{2(0)}\left(C_{-} \text {Density }\right)} \\
& +\frac{2 \tau_{3}}{x_{3(0)}\left(C \_ \text {Density }\right)}+\frac{2 \tau_{4}}{x_{4(0)}\left(C \_ \text {Density }\right)} \\
& +\frac{2 \tau_{5}}{x_{5(0)}\left(C \_ \text {Density }\right)} \text {. }
\end{aligned}
$$

The steps for the constant-SNR (minimizing die area) algorithm are as follows:

\section{Step 1) Calculate Null-Space Projection}

The direction of travel along the constant-constraint surface that produces the highest cost increase is calculated via the null-space projection $(P)$. This is the surface for which the noise remains constant in the chosen noise bandwidth.

$$
P=\vec{c}-\left[\mathbf{A}^{T}\left(\mathbf{A A}^{T}\right)^{-1} \mathbf{A}\right] \vec{c} .
$$

Step 2) Update Vector Function

The calculated projection is opposite of the desired direction (i.e., direction of decreasing cost), so the algorithm must take a small step in the $-P$ direction.

$\vec{x}_{k+1}=\vec{x}_{k}-w P, \quad$ where $w=$ weight.

The choice of the weight $w$ can be made arbitrarily small because our primary objective is to accurately arrive at the optimum values. In other words, we are not concerned with how quickly we arrive at the solution as long as it is accurate.

\section{Step 3) Reformulate Cost Function}

The new vector $\vec{x}_{k+1}$ is plugged into the linearized cost equations $\vec{c}$ and $D$, and a new linearized cost function results. This new cost function is used for the next iteration of the algorithm.

Steps 1 through 3 are repeated until the algorithm converges. The optimization generally converges after 20 iterations with a moderately conservative choice of weight (Step 2).

\section{CONSTANT-AREA OPtimization Algorithm}

In most filter design situations, a set area budget is less common than a set SNR design requirement. It is, however, interesting to measure the improvements gained by maintaining a constant die area and maximizing SNR for a given filter design. A simple redefinition of constraint and cost functions and a slightly adjusted algorithm (from the one presented in Section III) are all that is required to achieve this goal. Equations (5) and (7) are already linear functions representing noise and component area, respectively. For a constant-area optimization using this method, it is necessary that the area function be defined as the constraint (constant), and the noise function be defined as the cost.

In this new scenario, the constraint function is now the linearized function. This will require the addition of an extra step to the algorithm of Section III. While the constant-SNR (minimize area) optimization of Section III required Steps 1-3, this constant-area (maximize SNR) optimization will require one more step between Step 2 and Step 3. We will call this Step 2B.

Step 2B) Modify Updated Vector to Meet Constraint Surface When the nonlinear constraint from (6) is calculated using the new vector $\vec{x}_{k+1}$, it will have some offset from the desired noise level (the constraint which ought to be fixed) due to the linearization done on the equation used to calculate the constraint. A simple method to compensate for the offset is to scale the vector $\left(\vec{x}_{k+1}\right)$ back to the constraint surface. A corrected vector is calculated as

$$
\vec{x}_{k+1}(\text { new })=\vec{x}_{k+1} \cdot(1+\Delta)
$$

where $\Delta$ is the calculated linear offset.

The next step (Step 3) applies the new vector $\vec{x}_{k+1}$ (new) to the linearized constraint, from which a new linearized constraint function results. These steps (Steps 1,2, 2B, and 3) are repeated until the algorithm converges on a minimized cost value.

The major difference between the constant-noise/SNR algorithm and the constant-area algorithm is the presence of either a linear or linearized constraint function. In the case of the constant-area algorithm, once $\vec{x}$ is incremented to a new location (Step 2 of the algorithm), the vector produced is no longer on the constant-constraint surface. The overshoot or undershoot is then compensated for by a scaling that places the vector back on the constant-constraint surface. In the constant-SNR algorithm, the incremented vector does not miss the constraint surface because the constraint function is completely linear. 

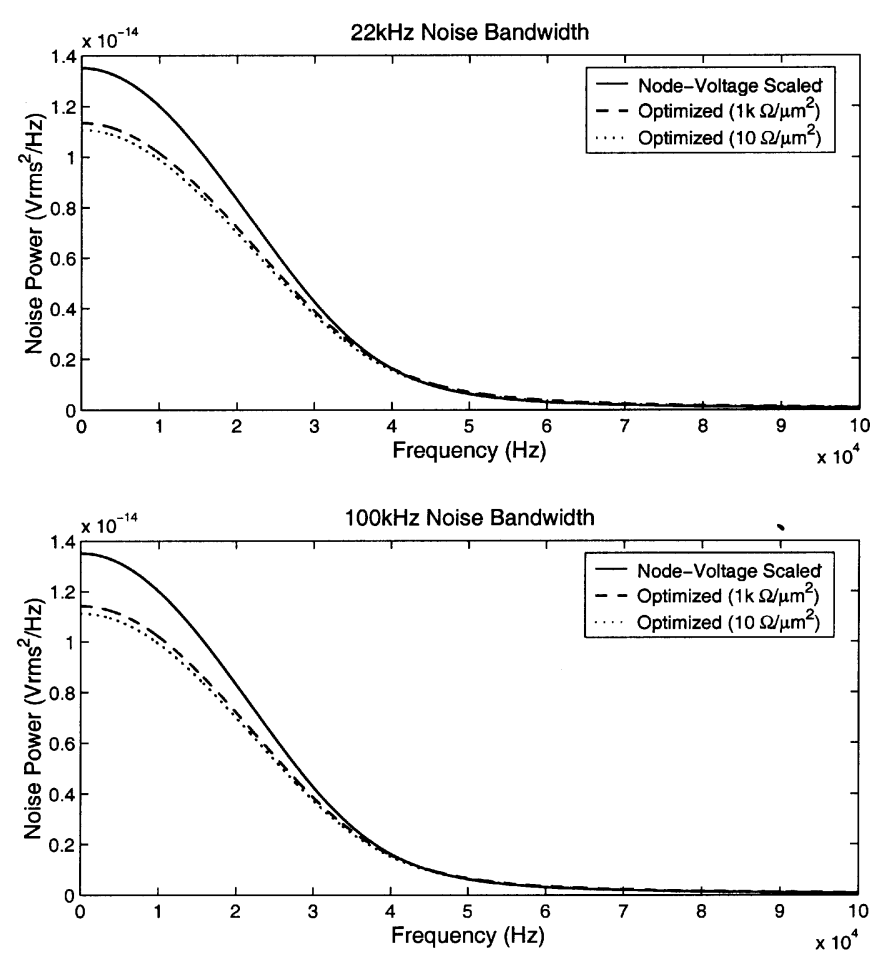

Fig. 2. Noise savings: fifth-order Bessel.

\section{OPTIMIZATION RESULTS}

The optimization process was done to each of the three filters for a variety of noise bandwidths. For the given design of $22-\mathrm{kHz}$ filter corner frequency, the optimization was applied to the ranges dc to $22 \mathrm{kHz}$ and dc to $100 \mathrm{kHz}$. Gains (squared) from each resistor to the output were integrated in the range for which they were to be optimized. Measurements of noise savings (and SNR improvement) were also integrated over the same frequency range. The capacitor density used for optimization was $1 \mathrm{fF} / \mu \mathrm{m}^{2}$. The optimization was completed for two resistor densities: $1 \mathrm{k} \Omega / \mu \mathrm{m}^{2}$ and $10 \Omega / \mu \mathrm{m}^{2}$.

For the $R \_$Density $=1 \mathrm{k} \Omega / \mu \mathrm{m}^{2}$ constant-SNR optimization with a dc-22 kHz noise bandwidth, area savings was $15.01 \%$ for the Bessel filter, $1.96 \%$ for the Chebychev filter, and $6.03 \%$ for the Butterworth filter. SNR improvement with the constant-area optimization was $0.71 \mathrm{~dB}$ for the Bessel filter, $0.09 \mathrm{~dB}$ for the Chebychev filter, and $0.27 \mathrm{~dB}$ for the Butterworth filter. For the dc-100 kHz noise bandwidth, the Chebychev filter had less area improvement and less SNR improvement (3.46\% and $0.15 \mathrm{~dB}$ ), the Bessel filter had slightly better area improvement and slightly better SNR improvement $(12.28 \%$ and $0.57 \mathrm{~dB})$, and the Butterworth filter had substantially larger improvements in both area and SNR $(21.63 \%$ and $1.06 \mathrm{~dB})$.

The same optimizations with a resistor density of $10 \Omega / \mu \mathrm{m}^{2}$ showed slightly higher SNR improvements than the $1 \mathrm{k} \Omega / \mu \mathrm{m}^{2}$ optimization. These increases occur because the optimization algorithm redistributes the extra area freed up by using higher density resistors to the capacitors.

Noise curves for the Bessel filter with noise bandwidths at 22 and $100 \mathrm{kHz}$ are shown in Fig. 2. Trends of the other two filter types, Chebychev and Butterworth, behave similarly. Improvement results of all optimizations for all three filter types and two resistor densities are shown in Tables III and IV. Figs. 3 and 4

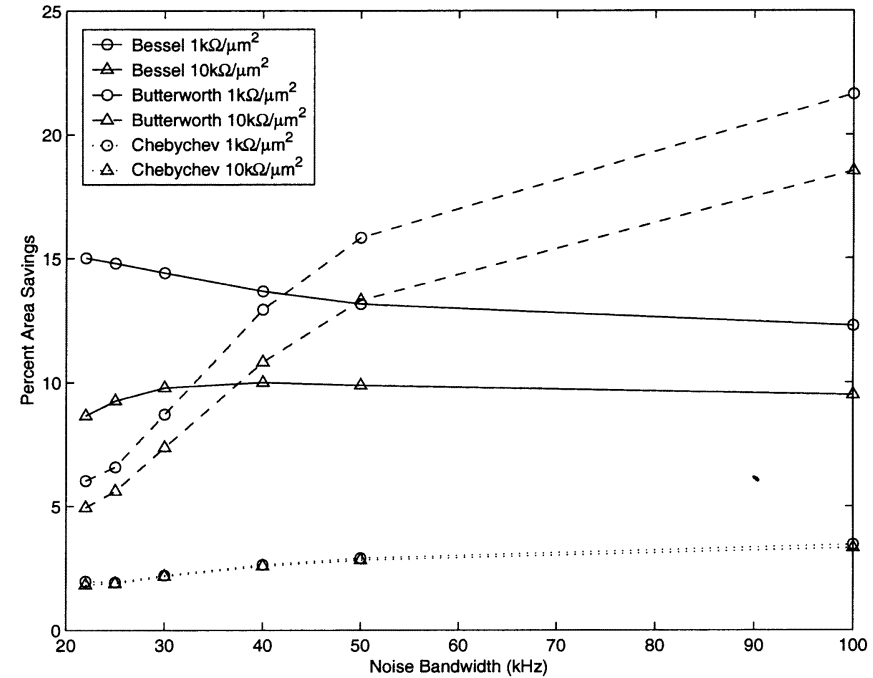

Fig. 3. Constant-SNR area savings trends.

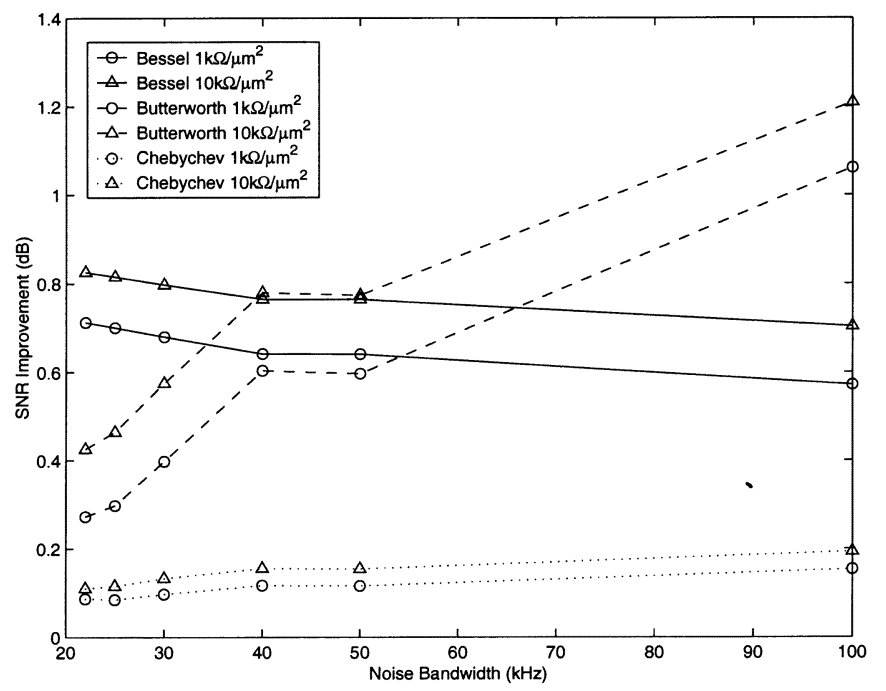

Fig. 4. Constant-area SNR improvement trends.

(data from Tables III and IV) show area savings and SNR improvement trends versus optimization bandwidths.

Final component spread values for the constant-area optimization are summarized in Table V. Generally speaking, the total component spread remains about the same for all three optimized filter types. The Bessel filter type, when optimized, has a larger resistor value spread (compared with capacitor spread), while the Chebychev and Butterworth filter types have relatively similar capacitor spread and resistor spread. The change of component spread is minimal and this should not negatively affect realizability (with regards to sensitivity) of the optimized filter.

\section{CONCLUSION}

Two algorithms for improvement of filter die area usage and SNR through linear programming have been presented. These improvements are accomplished without decreasing SNR design requirements or increasing die area budgets. Because the proposed method preserves the given frequency response and the filter topology/structure, it may be applied in addition to any other preferred optimization technique. The net improvement of the proposed technique is reduced IC die area for a given filter 
TABLE III

CONSTANT-SNR OPTIMIZATION AREA SAVINGS

\begin{tabular}{c||c|c||c|c||c|c}
\hline \multicolumn{1}{c||}{} & \multicolumn{2}{c||}{ Bessel } & \multicolumn{2}{c||}{ Chebychev } & \multicolumn{2}{c}{ Butterworth } \\
\hline Frequency Range & $1 \mathrm{k} \Omega / \mu m^{2}$ & $10 \Omega / \mu m^{2}$ & $1 \mathrm{k} \Omega / \mu m^{2}$ & $10 \Omega / \mu m^{2}$ & $1 \mathrm{k} \Omega / \mu m^{2}$ & $10 \Omega / \mu m^{2}$ \\
\hline \hline DC - 22k & $15.01 \%$ & $8.66 \%$ & $1.96 \%$ & $1.82 \%$ & $6.03 \%$ & $4.95 \%$ \\
\hline DC - 25k & $14.80 \%$ & $9.26 \%$ & $1.92 \%$ & $1.87 \%$ & $6.58 \%$ & $5.61 \%$ \\
\hline DC - 30k & $14.41 \%$ & $9.79 \%$ & $2.20 \%$ & $2.16 \%$ & $8.71 \%$ & $7.37 \%$ \\
\hline DC - 40k & $13.67 \%$ & $10.00 \%$ & $2.63 \%$ & $2.57 \%$ & $12.93 \%$ & $10.83 \%$ \\
\hline DC - 50k & $13.15 \%$ & $9.88 \%$ & $2.90 \%$ & $2.82 \%$ & $15.82 \%$ & $13.30 \%$ \\
\hline DC - 100k & $12.28 \%$ & $9.50 \%$ & $3.46 \%$ & $3.32 \%$ & $21.63 \%$ & $18.52 \%$ \\
\hline
\end{tabular}

TABLE IV

CONSTANT-AREA OPTIMIZATION SNR IMPROVEMENT (DB)

\begin{tabular}{c||c|c||c|c||c|c}
\hline \multicolumn{1}{c||}{} & \multicolumn{2}{c||}{ Bessel } & \multicolumn{2}{c||}{ Chebychev } & \multicolumn{2}{c}{ Butterworth } \\
\hline Frequency Range & $1 \mathrm{k} \Omega / \mu m^{2}$ & $10 \Omega / \mu m^{2}$ & $1 \mathrm{k} \Omega / \mu m^{2}$ & $10 \Omega / \mu m^{2}$ & $1 \mathrm{k} \Omega / \mu m^{2}$ & $10 \Omega / \mu m^{2}$ \\
\hline \hline DC - 22k & 0.7120 & 0.8253 & 0.0864 & 0.1096 & 0.2724 & 0.4255 \\
\hline DC - 25k & 0.7001 & 0.8150 & 0.0842 & 0.1148 & 0.2976 & 0.4643 \\
\hline DC - 30k & 0.6795 & 0.7972 & 0.0967 & 0.1323 & 0.3976 & 0.5751 \\
\hline DC - 40k & 0.6416 & 0.7644 & 0.1163 & 0.1548 & 0.6039 & 0.7793 \\
\hline DC - 50k & 0.6410 & 0.7640 & 0.1153 & 0.1540 & 0.5969 & 0.7737 \\
\hline DC - 100k & 0.5715 & 0.7033 & 0.1531 & 0.1933 & 1.0612 & 1.2098 \\
\hline
\end{tabular}

TABLE V

CAPACITOR AND RESISTOR SPREAD

\begin{tabular}{c||c|c||c|c||c|c}
\hline \multicolumn{1}{c||}{} & \multicolumn{2}{c||}{ Bessel } & \multicolumn{2}{c||}{ Chebychev } & \multicolumn{2}{c}{ Butterworth } \\
\hline & C Spread & R Spread & C Spread & R Spread & C Spread & R Spread \\
\hline \hline NV Scaled & $\mathbf{6 . 4 7}$ & $\mathbf{2 . 1 3}$ & $\mathbf{2 . 3 1}$ & $\mathbf{2 . 3 0}$ & $\mathbf{4 . 3 0}$ & $\mathbf{2 . 0 0}$ \\
\hline \hline $22 \mathrm{kHz}\left(1 \mathrm{k} \Omega / \mu m^{2}\right)$ & 1.81 & 7.61 & 1.94 & 2.64 & 2.65 & 3.35 \\
\hline $50 \mathrm{kHz}\left(1 \mathrm{k} \Omega / \mu m^{2}\right)$ & 1.97 & 6.99 & 1.41 & 2.04 & 1.34 & 3.14 \\
\hline $100 \mathrm{kHz}\left(1 \mathrm{k} \Omega / \mu m^{2}\right)$ & 2.09 & 6.58 & 1.32 & 2.17 & 1.27 & 3.72 \\
\hline \hline $22 \mathrm{kHz}\left(10 \Omega / \mu m^{2}\right)$ & 2.12 & 6.50 & 1.84 & 2.20 & 2.28 & 3.18 \\
\hline $50 \mathrm{kHz}\left(10 \Omega / \mu m^{2}\right)$ & 2.10 & 6.54 & 1.40 & 2.05 & 1.40 & 3.09 \\
\hline $100 \mathrm{kHz}\left(10 \Omega / \mu m^{2}\right)$ & 2.19 & 6.27 & 1.31 & 2.17 & 1.29 & 3.54 \\
\hline
\end{tabular}

design and SNR. An alternative approach would yield increased SNR for the fixed die area used. Both algorithms guarantee improvement for a chosen noise bandwidth. The die area savings can be as much as $21.6 \%$ and SNR improvement as high as $1.2 \mathrm{~dB}$ for some filter types.

Improvements found with different frequency-band weighting techniques such as "A-Weighting" (common in audio applications) would be an interesting extension to this work. A more circuit-specific extension of this work would be to consider the operational amplifier noise, and the component mismatches (sensitivity) in the development of constraint and cost functions. It is anticipated that low- $Q$ filters, such as Butterworth where it benefits most from the proposed optimization, would not be significantly affected by these mismatches. Further areas of study related to this topic are noise optimization in switched-capacitor filters and other circuit types.

\section{ACKNOWLEDGMENT}

The authors would like to thank the anonymous reviewers for their comments which have been incorporated into the final version of this manuscript.

\section{REFERENCES}

[1] U. Moon and B. Song, "Design of a low-distortion 22-kHz fifth-order Bessel filter," IEEE J. Solid-State Circuits, vol. 28, pp. 1254-1264, Dec. 1993.

[2] S. Nordebo and Z. Zhang, "Semi-infinite linear programming: A unified approach to digital filter design with time- and frequency-domain specification," IEEE Trans. Circuits Syst. II, vol. 46, pp. 765-775, June 1999.

[3] N. Damera-Venkata and B. Evans, "An automated framework for multicriteria optimization of analog filter designs," IEEE Trans. Circuits Syst. II, vol. 46, pp. 981-990, Aug. 1999.

[4] R. Mackay and A. Sedra, "Generation of low-sensitivity state-space active filters," IEEE Trans. Circuits Syst., vol. CAS-27, pp. 863-870, Oct. 1980.

[5] G. Groenewold, "The design of high dynamic range continuous-time integratable bandpass filters," IEEE Trans. Circuits Syst., vol. 38, pp. 838-852, Aug. 1991.

[6] W. Ki and G. Temes, "Optimal capacitance assignment of switched-capacitor biquads," IEEE Trans. Circuits Syst. I, vol. 42, pp. 334-342, June 1995.

[7] D. Johns, W. Martin, and A. Sedra, "Orthonormal ladder filters," IEEE Trans. Circuits Syst., vol. 36, pp. 337-343, Mar. 1989.

[8] A. Zverev, Handbook of Filter Synthesis New York, Wiley, 1967.

[9] A. Williams and F. Taylor, Electronic Filter Design Handbook. New York: McGraw-Hill, 1988.

[10] R. Schaumann, M. Ghausi, and K. Laker, Design of Analog Filters. Englewood Cliffs, NJ: Prentice-Hall, 1990.

[11] S. Gilbert, Linear Algebra and its Applications, 3rd ed. San Diego, HBJ, 1988. 